AperTO - Archivio Istituzionale Open Access dell'Università di Torino

Theoretical and experimental determination of the absorption and emission spectra of a prototypical indolenine-based squaraine dye

This is a pre print version of the following article:

Original Citation:

Availability:

This version is available http://hdl.handle.net/2318/157809

since 2016-11-30T14:14:46Z

Published version:

DOI:10.1039/C3CP54298J

Terms of use:

Open Access

Anyone can freely access the full text of works made available as "Open Access". Works made available under a Creative Commons license can be used according to the terms and conditions of said license. Use of all other works requires consent of the right holder (author or publisher) if not exempted from copyright protection by the applicable law. 


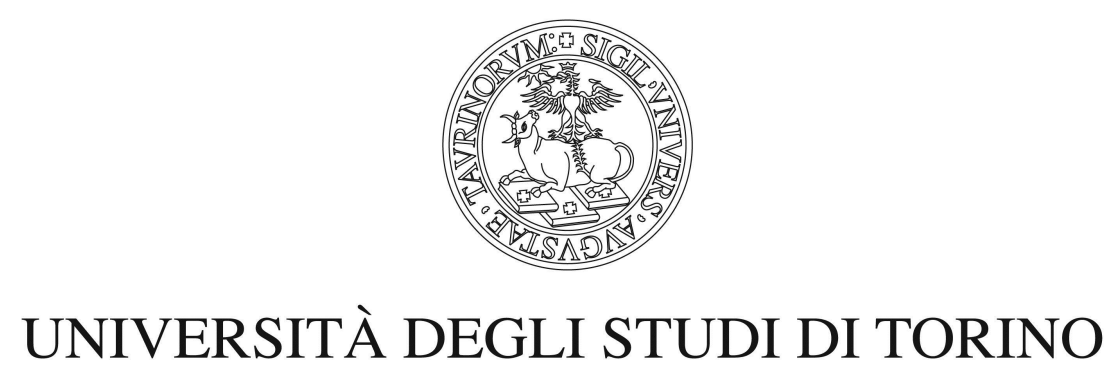

This is an author version of the contribution published on:

Raffaele Borrelli, Silvano Ellena, and Claudia Barolob. Theoretical and Experimental Determination of the Absorption and Emission Spectra of a Prototypical Indole-based Squaraine Dye . Physical Chemistry Chemical Physics, 16, 2390, 2014, 10.1039/C3CP54298J.

The definitive version is available at:

http://pubs.rsc.org/en/content/articlelanding/2014/cp/c3cp54298j 


\title{
Theoretical and Experimental Determination of the Absorption and Emission Spectra of a Prototypical Indole-based Squaraine Dye
}

\author{
Raffaele Borrelli*a ${ }^{*}$ Silvano Ellena ${ }^{b, c}$ and Claudia Barolo ${ }^{b, c}$
}

November 21, 2013

\begin{abstract}
The spectroscopy of a prototypical indole-based squaraine dye is analysed theoretically using state-of-the-art methodologies for the simulation of spectral lineshapes, and experimentally using optical absorption and emission spectroscopies. Density functional theory and its time-dependent extension are used to determine the stability of several conformers, to compute their excitation energies, equilibrium geometries and vibrational frequencies, both in the ground and in their first excited singlet state. Finally the generating function approach is used to simulate the vibronic lineshape of the low energy valence $\pi \pi^{*}$ excitation and emission spectra. Solvent effects are also computed and discussed by using the polarizable continuum model. The developed model correctly reproduces the main spectral features of the squaraine, and allows to identify the vibrational motions which mostly contribute to the observed lineshape.
\end{abstract}

${ }^{a}$ Department of Agricultural, Forestry and Food Science, University of Torino, Via Leonardo da Vinci 44, I-10095 Grugliasco, Italy; Tel: 0116708551; E-mail: raffaele.borrelli@unito.it

${ }^{b}$ Department of Chemistry, University of Torino, Via Pietro Giuria 7, I-10125 Torino, Italy

${ }^{c}$ Department of Chemistry and NIS Interdepartmental Centre of Excellence, University of Torino, Via Pietro

Giuria 7, I-10125 Torino 


\section{Introduction}

The calculation and analysis of absorption and emission spectra of molecules from first principles is a fundamental problem of modern physical chemistry, with a widespread range of applications, extending from the understanding of the basic aspects of light-matter interaction to the development of organic dyes with tailored spectroscopic properties. ${ }^{1,2}$

Fast growing research fields such as organic LED technology ${ }^{3}$ and dye-sensitized solar cells (DSSC), ${ }^{4}$ would strongly benefit from the application of robust and easy-to-use methodologies for the simulation of spectral lineshapes of organic dyes. The research in this field is quickly growing $^{5-21}$ (see also Borrelli et al. ${ }^{22}$ for a recent review) but standardize procedures have not yet been established, and specific computational strategies must be carefully designed and require validation and testing on several class of molecules. ${ }^{23-26}$ In this paper we apply state of the art tools of computational spectroscopy to simulate the absorption and emission lineshapes of squaraine dyes in solution.

The squaraine class of molecules is well known for its spectroscopic characteristic. The photochemical and photophysical properties of these dyes have been studied extensively ${ }^{27-31}$ because of their many technological applications in copiers and laser printers, ${ }^{32-34}$ organic optical disks ${ }^{35}$ and organic solar cells ${ }^{36-42}$. The general structure of symmetric squaraines can be described as a D-A-D system, where $\mathrm{D}$ is an electron donor and $\mathrm{A}$ is the $\mathrm{C}_{4} \mathrm{O}_{2}$ squaric ring, which plays an electron acceptor role. They are characterized by a very strong absorption due to a valence $\pi \pi^{*}$ excitation in the long wavelength region. In most cases a high energy transition with much lower intensity is also detected but its origin has not been deeply investigated.

Among others, indole-based squaraines have been extensively studied in the last few years mainly because of their very high photostability that makes them perfect candidates for several applications, for example in DSSC, ${ }^{39,43-45}$, protein labeling, and as photosensitizers. ${ }^{46,47}$ Retting and Gude ${ }^{48}$ have shown that indole-based squaraines can undergo a quite complex photoisomerization process upon excitation into the $S_{1}\left(\pi \pi^{*}\right)$ state, which would explain the observed temperature dependence of fluorescence spectra and yield. Quite recently a detailed experimental study of both photoisomerization and ground state isomerization processes in several indole squaraines has been proposed by de Miguel et. al strongly deepening the understanding of the 
complex photodynamics of such molecules. ${ }^{49,50}$ Dirk et al. ${ }^{31}$ have investigated the non-linear optical properties of a simple bis(indole)-squaraine (ISQ) interpreting the absorption spectrum as a result of the superposition of the absorption profiles of two conformational isomers. In their work, however, the contribution of the vibrational transitions to the absorption lineshape is completely neglected.

The investigation reported in this paper encompasses both a theoretical and an experimental study of the spectroscopy of ISQ. We present an in-depth theoretical analysis of the excited electronic states of bis(indole)-squaraine, and compute the lineshape associated with $\mathrm{S}_{1}\left(\pi \pi^{*}\right) \leftarrow \mathrm{S}_{0}$ electronic transition using the generating function (GF) formalism. A targeted direct calculation of a few Franck-Condon integrals for the correct assignment of the vibronic bands is also performed. With this approach we have been able to identify the vibrational modes which are responsible for the vibronic structure of the absorption spectrum.

The experimental investigation comprises both the synthesis of ISQ based on a newly developed procedure ${ }^{51}$ as well as its characterization by means of absorption and emission spectroscopies.

\section{Experimental Section}

\subsection{Synthesis and characterization}

All solvents and reagents were purchased from commercial sources and used as received without further purification, unless otherwise stated. Biotage MW synthesizer (Model: Initiator EXP EU 355301) was used for microwave syntheses. TLC were performed on silica gel 60 F254 plates using dichloromethane (DCM) and methanol (90:10) as eluents. ESI-MS spectra (positive ions) were recorded using a LCQ Deca XP plus spectrometer (Thermo), with electrospray interface and ion trap as mass analyzer. The flow injection effluent was delivered into the ion source using nitrogen as sheath and auxiliary gas. 1H and 13C NMR spectra were recorded on a Brucker Avance 200 or on a Jeol EX-400 and calibrated to the residual solvent peak. For all spectroscopic measurements, spectroscopic grade solvents were used. UV/Vis/NIR spectra were recorded on Shimadzu PharmaSpec UV-1700 and fluorescence spectra were recorded with a Perkin-Elmer LS55. Absorption spectra were collected using tetrahydrofurane (THF), as sol- 
vent, in the concentration range $10^{6} 10^{5} \mathrm{M}$. The range for fluorescence emission recording was from 625 to $900 \mathrm{~nm}$. The excitation wavelength was set at $615 \mathrm{~nm}$. The squarylium dye ISQ

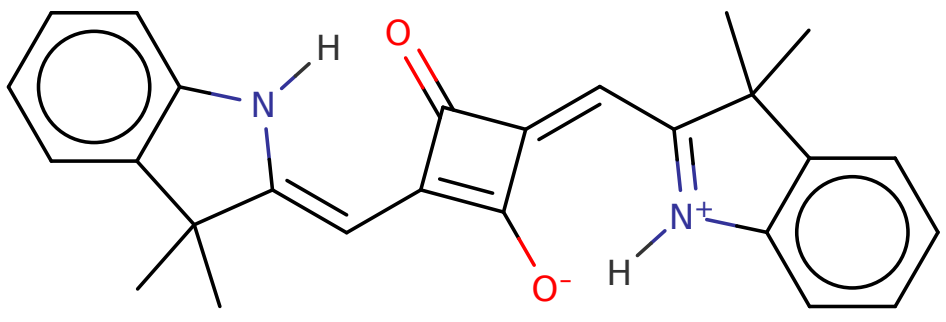

has been synthesized as follows. ${ }^{31,51}$ Squaric acid (114 mg, $1 \mathrm{mmol}$ ) and 2,3,3-trimethylindolenine $(318.46 \mathrm{mg}, 2 \mathrm{mmol})$ in a mixture of toluene $(2 \mathrm{~mL})$ and $\mathrm{n}$-butanol $(2 \mathrm{~mL})$ were heated at 160 $\mathrm{C}$ for 20 minutes in a sealed microwave vial. The reaction mixture was cooled to room temperature and the solvents were removed by rotary evaporation. The residue was treated with diethyl ether $(10 \mathrm{~mL})$ and the obtained powder was filtered and washed few times. A pure dark green solid powder was obtained. Yield: $41 \%(242 \mathrm{mg})$. NMR and ESI-MS analyses are in agreement with the data reported in literature.

\subsection{Experimental results}

NMR spectra put in evidence the existence of two isomers with quite similar population at room temperatures (see infra), confirming the results already reported by Dirk et al. ${ }^{31}$

The measured absorption and emission profiles of ISQ in THF are reported in figure 1. The absorption spectrum shows a very intense band between 550 and $700 \mathrm{~nm}$, with two main components, the most intense peaked at $659 \mathrm{~nm}$ and a shoulder around $615 \mathrm{~nm}$. A third quite flat shoulder at around $566 \mathrm{~nm}$ can be revealed after fitting the spectrum with a linear combination of Gaussian profiles. The three components have an average spacing of $1200-1400 \mathrm{~cm}^{-1}$ which seems to indicate that they are associated to a vibronic progression.

At higher energy the spectrum becomes more complex to analyze since there is no clear-cut separation between different electronic transitions. At least two components can be identified peaked around $280 \mathrm{~nm}$ and $360 \mathrm{~nm}$, though a non-negligible absorption is also evident around $420 \mathrm{~nm}$. See infra for a detailed discussion and assignment of the electronic transitions. 
The fluorescence spectrum is similar to those already reported for similar systems. ${ }^{48}$ The Stoke shift is quite small as already reported for many squaraines. We notice that the fairly intense shoulder which appears in the absorption profile is less pronounced in the emission spectrum. Possible causes of this asymmetry will be discussed later. In table 1 the molar extinction coefficient, the absorption and emission maxima and their theoretical (see infra) estimate are reported.

\section{Theoretical Methodology}

\subsection{Theory of lineshapes}

Absorption and emission molecular lineshapes can be computed using the so-called generatingfunction (GF) theory. ${ }^{52}$ Details on the derivation and implementation of the GF formalism can be found elsewhere, ${ }^{53}$ here we summarize the main results. The lineshape of a radiative transition from a manifold of thermally populated vibronic states $\left|v_{g}\right\rangle$ to a manifold of excited vibronic states $\left|v_{e}\right\rangle$ is:

$$
I(\omega, T)=\left(\hbar Z_{v}\right)^{-1} \sum_{\alpha=x, y, z v_{g}, v_{e}} e^{-\beta E\left(v_{g}\right)}\left|\left\langle v_{g}\left|\mu_{\alpha}^{g e}\right| v_{e}\right\rangle\right|^{2} \delta\left(\omega+E\left(v_{g}\right)-E\left(v_{e}\right)-\Delta E_{e g}\right)
$$

where $E\left(v_{g}\right)$ and $E\left(v_{e}\right)$ are the energies of the vibrational states in the initial and final electronic state, $|g\rangle$ and $|e\rangle, \Delta E_{e g}$ is the electronic energy difference, $\mu_{\alpha}^{g e}$ is the $\alpha$-th component of the electric transition dipole moment, and $\beta=1 / k T$, with $k$ the Boltzmann constant. Alternative formulations have also been provided by other authors, see for example ${ }^{54-56}$.

Following the seminal works of $\mathrm{Lax}^{57}$ and Kubo, ${ }^{52}$ the spectral distribution $I(\omega, T)$ can be conveniently rewritten as:

$$
I(\omega, T)=2 \pi \int_{-\infty}^{+\infty} e^{-i\left(\omega+\Delta E_{e g}\right) \tau} f(\tau) d \tau
$$

where

$$
f(\tau)=\operatorname{Tr}\left\{\mu e^{-i \tau H_{e}} \mu e^{-(\beta-i \tau) H_{g}}\right\} / \operatorname{Tr}\left\{e^{-\beta H_{g}}\right\}
$$

and $H_{g}, H_{e}$ are the Hamiltonian operators of the initial (ground) and final (excited) electronic states respectively. If the latter ones are expressed in harmonic approximation, and if the transition dipole moment is taken as constant (Condon approximation) the correlation function $f(\tau)$ 
assumes the following closed form: ${ }^{52}$

$$
f(\tau)=|\mu|^{2}[\operatorname{det} \Phi]^{-1 / 2} \exp \left[-\tilde{\Delta} \mathbf{T}_{g}\left(\mathbf{T}_{g}+\mathbf{T}_{e}\right)^{-1} \mathbf{T}_{e} \Delta\right]
$$

where

$$
\begin{aligned}
\mathbf{T}_{g} & =\omega_{g} \tanh \left[(\beta-i \tau) \omega_{g} / 2\right] ; \quad \mathbf{T}_{e}=\tilde{\mathbf{J}} \omega_{e} \tanh \left(i \tau \omega_{e} / 2\right) \mathbf{J} \\
\mathbf{C}_{g} & =\omega_{g} / \tanh \left[(\beta-i \tau) \omega_{g} / 2\right] ; \quad \mathbf{C}_{e}=\tilde{\mathbf{J}} \omega_{e} / \tanh \left(i \tau \omega_{e} / 2\right) \mathbf{J} \\
\Phi & =\left[2 \sinh \left(\beta \omega_{g} / 2\right)\right]^{-2} \omega_{g}^{-1} \sinh \left[(\beta-i \tau) \omega_{g}\right]\left(\mathbf{T}_{g}+\mathbf{T}_{e}\right)\left(\mathbf{C}_{g}+\mathbf{C}_{e}\right) \omega_{e}^{-1} \sinh \left(i \tau \omega_{e}\right) \\
\Delta & =-\mathbf{J}^{-1} \mathbf{K} ;
\end{aligned}
$$

Here the Duschinsky matrix $\mathbf{J}$ and the displacement vector $\mathbf{K}$ are define through the affine transformation between the normal modes of the ground $\left(\mathbf{Q}_{g}\right)$ and of the excited state $\left(\mathbf{Q}_{e}\right)$

$$
\mathbf{Q}_{e}=\mathbf{J} \mathbf{Q}_{g}+\mathbf{K} .
$$

The GF formulation is very efficient for numerical treatments because the calculation of $I(\omega, T)$ can be recast into a discrete Fourier transform problem. This approach has been recently applied to the simulation of spectral lineshapes of large molecules ${ }^{58}$ as well as to the determination of electron-transfer rates with positive results. ${ }^{18,19,53}$

\subsection{Computational details}

The ground state equilibrium geometry and the normal modes of vibration of the indole squaraine have been computed using density functional theory (DFT), employing the hybrid PBE0 functional ${ }^{59}$ with the $6-31+\mathrm{G}(\mathrm{d}, \mathrm{p})$ Pople-type basis set. Satisfactory results have already been obtained with the above approach for other large pigments such as chlorins. ${ }^{58}$ Equilibrium geometries and normal modes of the two lowest excited states have been calculated by the timedependent density functional theory (TD-DFT) approach, using the same functional and basis set of the ground state calculations. Vertical excitation energies have also been computed with the same methods. Bulk solvent effects have been included using the polarizable continuum model (PCM).

DFT and TD-DFT calculations have been performed using the Gaussian09 software package. ${ }^{60}$ The excited state Hessian is obtained by numerical differentiation of the gradients with a step of $0.05 \AA$. 
The Duschinsky matrix $\mathbf{J}$ and the displacement vector $\mathbf{K}$ have been computed using the internal coordinate representation of normal modes. ${ }^{5,7,9,53,61-63}$ The generating function technique has been implemented in the MolFC software ${ }^{64}$. The assignment of the vibronic peaks to a specific transition is performed by first computing the entire lineshape, then searching for vibronic states in the selected energy range, and computing the associated FC integrals.

\section{Electronic spectrum of ISQ}

ISQ can exist in several isomeric forms as sketched in figure 2. The relative stability of the isomers has been determined using DFT/PCM calculations and is reported in figure $2 \mathrm{in} \mathrm{kcal} / \mathrm{mol}$. The isomer labeled as ISQ6 is the most stable and is almost isoenergetic with ISQ4. All the other isomers have a much higher energy and their Boltzmann populations at room temperature can be safely neglected. The results are in qualitative agreement with previous data reported by Dirk et al. who found ISQ4 to be slightly more stable than ISQ6. ${ }^{31}$ The energy differences between the isomers are very likely due to the different number of intramolecular hydrogen bonds. It appears thus reasonable to assume that in a non-protic solvent the squarain can exist as a mixture of the species ISQ4 and ISQ6 with very similar Boltzmann populations. Following the above conclusions the absorption and emission lineshapes can be described as a combination of the electronic spectra of the two isomers.

The ground state equilibrium geometries of the ISQ6 and ISQ4 isomers computed at DFT level are predicted to belong to the $C_{2 h}$ and $C_{2 v}$ symmetry point groups respectively, with the two heteroaromatics and the squaric ring coplanar. Therefore, in ISQ6 only vertical transitions to electronic states belonging to the $A_{u}$ and $B_{u}$ representations are allowed, the ground state being totally symmetric, while in ISQ4 transitions to $A_{1}, B_{1}$ and $B_{2}$ states are allowed. Table 2 shows the allowed vertical excitations computed at TD-DFT level up to $260 \mathrm{~nm}$ for both isomers, while figure 3 shows the position and the relative intensity (in THF) of the transitions together with the experimental absorption.

The computed lowest energy transitions of ISQ4 and ISQ6 in THF fall at $566 \mathrm{~nm}$ and 564 $\mathrm{nm}$ respectively, and can be associated with the experimentally observed absorption between $550 \mathrm{~nm}$ and $700 \mathrm{~nm}$. The computed excitation energies of the two isomers to the first excited 


\subsection{Franck-Condon profile of the $S_{1} \leftarrow S_{0}$ transition $\quad$ ELECTRONIC SPECTRUM OF ISQ}

singlet differ by only $0.01 \mathrm{eV}$, and in both cases the state is described by a HOMO-LUMO $\left(\pi \pi^{*}\right)$ excitation. The molecular orbitals which characterize the transition are shown in figure 4. We notice that the aromatic rings do not contribute significantly to these two orbitals which are mainly localized on the $\mathrm{C}_{4} \mathrm{O}_{2}$ ring and on the polymethine-like chain.

For both isomers the computed allowed transitions at higher energies are predicted to be of much lower intensity. The number of electronic transitions in the ISQ4 isomer is higher than that of ISQ6, and this is clearly due to the different symmetry of the two systems. The broad and non-structured nature of the absorption, and the large number of computed electronic transitions make the analysis of the high energy region of the spectrum quite difficult. The absorption around 400-350 $\mathrm{nm}$ can be safely attributed to the first $A_{1}$ and $B_{1}$ singlets of the ISQ4 isomer, since ISQ6 does not show any significant transition in that region. The origin of the band below $330 \mathrm{~nm}$ is much more problematic. As evident from figure 3 several transitions of both isomers appear to contribute to the absorption in this spectral region. The state $5^{1} A_{1}$ of

ISQ4 has the largest oscillator strength (0.33) and, together with the states $4^{1} A_{1}$ and $5^{1} B_{2}$, and the states $4^{1} B_{u}$ and $6^{1} B_{u}$ of ISQ6 provide most of the computed intensity in this energy region.

\subsection{Franck-Condon profile of the $S_{1} \leftarrow S_{0}$ transition}

In order to simplify the calculation of the electronic lineshapes associated with the transition to the first excited singlet we have introduced a model system in which the methyl groups of the molecule have been substituted by hydrogen atoms. That is expected to introduce only minor differences with the original model mainly related to the broadening of the vibronic lines, associated with the presence of low frequency vibrations of the methyl groups.

Equilibrium geometry computations at TD-DFT level predict that in the first excited singlet the isomers ISQ6 and ISQ4 have $C_{2 h}$ and $C_{2 v}$ symmetry respectively thus maintaining the symmetry of the ground state.

The structural changes that follow the electronic excitations, which are at the origin of the observed vibronic progressions, are quite small. Indeed, excitation from $S_{0}$ to $S_{1}$ induces a variation in the bond length alternation of the polymethine-like chain, in strict analogy with what happens in cyanines. ${ }^{65}$ The largest change in bond length is about $0.017 \AA$ in THF and $0.015 \AA$ in vacuo. The largest variation of bending coordinate is only 1.6 degrees in THF 


\subsection{Franck-Condon profile of the $S_{1} \leftarrow S_{0}$ transition $\quad$ ELECTRONIC SPECTRUM OF ISQ}

and 1.7 degrees for the isolated molecule. The computed effect of solvent on the equilibrium geometries of the two states is absolutely marginal, as expected from a valence $\pi \pi^{*}$ excitation in a low-polarity solvent.

The dimensionless displacements of normal vibrations are reported in table 4. ISQ6, has 40 totally symmetric $\left(a_{g}\right)$ vibrations with a non-zero shift. Calculations in THF predict that the most displaced modes correspond to two low frequency vibrations falling at $87 \mathrm{~cm}^{-1}$ and 202 $\mathrm{cm}^{-1}$ with an absolute value of the dimensionless shift of 0.59 and 0.70 respectively. These shifts become 0.665 and 0.633 without solvent effects. The two vibrations can be described as superpositions of bending and stretching type motions of the two indolenin moieties relative to the squaric ring.

ISQ4 has 40 totally symmetric $\left(a_{1}\right)$ vibrations, and their dimensionless displacements are reported in tables 3. The most displaced modes in this case fall at $40 \mathrm{~cm}^{-1}$ and $217 \mathrm{~cm}^{-1}$, with the former having having quite a large value of about 1.3 in THF.

For both isomers, vibrations with frequencies ranging from $1100 \mathrm{~cm}^{-1}$ to $1800 \mathrm{~cm}^{-1}$ have much smaller displacements, of the order of 0.1-0.25. Non negligible Duschinsky effects can be found only between modes labeled as 23, 24, 25 and 26, in ISQ6 and 22, 23, 24 and 25 in ISQ4. See infra for the importance of Duschinsky effects in the overall appearance of the spectrum.

The computed absorption spectra of ISQ6 and ISQ4 at room temperature are shown in figures 5 and 6 . All spectra are obtained by convoluting the result of the generating function calculation with a Gaussian lineshape function having a full width at half maximum of 300 $\mathrm{cm}^{-1}$. This is a typical value used for line broadening in condensed phase. ${ }^{66}$ To better understand the origin of the spectral shape the main vibronic peaks of the $0 \mathrm{~K}$ spectrum are also shown. All the stick spectra refer to the isolated isomer without bulk solvent effects.

The absorption spectrum of isolated ISQ6 show a fairly intense band close to the origin, and a secondary absorption about five times smaller shifted by approximately $1500 \mathrm{~cm}^{-1}$ from the main peak. The analysis of the Franck-Condon stick spectrum at $0 \mathrm{~K}$, allows to disentangle the major contributions to the observed spectral features. The spectrum shows a very intense 0-0 transition, the computed FC factor being 0.43; two absorption peaks at $87 \mathrm{~cm}^{-1}$ and 205

$\mathrm{cm}^{-1}$ are clearly visible and are due to $0-1$ transitions of the low frequency totally symmetric vibrations. A number of lines with lower intensity, associated with fundamental transitions, are 


\subsection{Franck-Condon profile of the $S_{1} \leftarrow S_{0}$ transition $\quad$ ELECTRONIC SPECTRUM OF ISQ}

clearly evident in the regions around $500 \mathrm{~cm}^{-1}$ and $1500 \mathrm{~cm}^{-1}$. The peaks at $1255 \mathrm{~cm}^{-1}$ and $1592 \mathrm{~cm}^{-1}$ are the most intense transitions in the medium frequency region, and are associated to a squaric ring deformation and to a skeleton vibration respectively. The dimensionless displacement of vibration falling at 1592 is 0.244 and originates mainly from the small variations in the $\mathrm{C}-\mathrm{C}$ bond length which follow the electronic transition. Transition at energies lower that the 0-0 peak are hot-bands arising from a non negligible Boltzmann population of the two totally symmetric low energy vibrations falling at $88 \mathrm{~cm}^{-1}$ and $203 \mathrm{~cm}^{-1}$ in the electronic ground state. Similar analyses hold for the spectral lineshape computed at DFT/TD-DFT level in THF, with only minor differences due to changes in the vibrational frequencies of the ground and excited electronic states and in the normal mode displacements.

The spectrum of the ISQ4 isomer, see figure 6 has a strict resemblance with that of ISQ6. tough the secondary should appears split into two contributions, and a non-negligible absorption is evident in the high energy region between $3000 \mathrm{~cm}^{-1}$ and $4000 \mathrm{~cm}^{-1}$. The analysis of the stick spectrum reveals that in this case the $0-0 \mathrm{FC}$ factor reduces to 0.25 , suggesting a more significant vibronic activity of ISQ4; the peaks close to the 0-0 transition are assigned to a progression of the lowest frequency vibration at $40 \mathrm{~cm}^{-1}$ which exhibits a very large displacement upon excitation. Unlike ISQ6, in the region between $1000 \mathrm{~cm}^{-1}$ and $1800 \mathrm{~cm}^{-1}$ the spectrum of ISQ4 appears denser with a significant number of lines. The spacing between the lines is narrower mainly because of the presence of combination bands with the low frequency vibration at $40 \mathrm{~cm}^{-1}$. The absorption at high energies (3000-4000 $\mathrm{cm}^{-1}$ ) arises from double excitations and combinations bands of the vibrations in the range $1500-1600 \mathrm{~cm}^{-1}$, more specifically its intensity is a result of a strong Duschinsky effect between the totally symmetric modes numbered as 29, 30, 31 and 32 in table 3. Again, the spectrum obtained when solvent effects are taken into account shows very minor differences with that computed for the isolated molecule.

The computed emission spectra of ISQ4 and ISQ6 are reported in figures 7 and 8. The spectra are almost the mirror image of the absorption, as expected from a system in which the variations of vibrational frequencies and normal mode directions upon excitation are quite small. The origin of the low intensity side band is analogous to that discussed for the absorption spectrum, i.e. it is mainly the result of fundamental transitions involving vibrations in the range $1000-1500 \mathrm{~cm}^{-1}$. 
Figure 9 shows the computed absorption and emission spectra of ISQ superimposed with the experimental data. The experimental data are transformed to normalized lineshapes for a better comparison with the computations. ${ }^{67,68}$ The spectra have been obtained as a superposition of the computed intensities of the two isomers with coefficients proportionals to their fractional Boltzmann population. The absorption and emission spectra have been shifted to lower energies by $2500 \mathrm{~cm}^{-1}$ and $280 \mathrm{~cm}^{-1}$ with respect to the computed vertical transition energy respectively. The intensities of both spectra have been scaled to match the maximum intensity of the observed lineshape.

From a quick comparison with the experimental data it is evident that DFT calculations provide a fairly good description of the overall absorption lineshape. Indeed, both the main absorption band as well as the small side band originating from fundamental vibronic transitions are correctly reproduced in relative position, width and intensity.

The simulation of the fluorescence spectrum is not yet completely satisfactory. Indeed, the main band with higher intensity is reasonably reproduced, however the relative intensity of the computed vibronic shoulder around $740 \mathrm{~nm}$ is clearly much higher than the experimental counterpart. Furthermore, the experimental spectrum falls off more rapidly at longer wavelengths.

Several factors can contribute to such a discrepancy. Apart from unavoidable accuracy problems in the computational methodology our model does not take into account the effects of the excited state photoisomerization on the electronic spectrum. ${ }^{48,69}$ It is thus not surprising that our model, though considering most of the vibrational motion coupled to the electronic transition, cannot quantitatively describe the electron spectroscopy of ISQ.

\section{Conclusions}

The spectral features of a prototypical indole squaraine have been analysed by means of DFT/TDDFT with and without solvent effects, combined with the recently implemented generatingfunction theory of molecular lineshapes.

Concerning the $S_{1} \leftarrow S_{0}$ electronic transition, the model proposed in this paper provide a semi-quantitative agreement with the experimental absorption spectrum. The side band of the spectrum which extends from $1000 \mathrm{~cm}^{-1}$ to $1500 \mathrm{~cm}^{-1}$ is originated by fundamental transitions 
of several vibrational modes of the molecule, mainly involving stretchings and bending motions of the molecular skeleton.

Unlike the absorption, the emission spectrum is only qualitatively reproduced. Apart from factors related to the computational accuracy, the shape of the measured fluorescence spectrum can be strongly influenced by the non-radiative decay induced by the photoisomerization in

the $S_{1}$ state. ${ }^{48,49}$ In such cases a quantitative agreement can only be obtained by using a more sophisticated model including the non-adiabatic coupling between the two electronic states. ${ }^{5,70}$

The assignment of the electronic transitions in the high energy region of the absorption spectrum poses several problems. According to TD-DFT results a significant number of transitions fall in that spectral region though only a few of them have a significant oscillator strength. In particular, we have identified 5 electronic states, 3 belonging to ISQ4 and 2 to ISQ6, that have a strong absorption from $450 \mathrm{~nm}$ to $250 \mathrm{~nm}$. Work is in progress to perform higher level calculations to support and validate TD-DFT results.

Finally we notice that DFT/TD-DFT methods provide quite a reliable description of the ground and excited state geometry and vibrations of this class of molecules, and the inclusion of bulk solvent effects using PCM has only a marginal impact on the computed lineshape of the $\mathrm{S}_{1} \leftarrow \mathrm{S}_{0}$ transition.

\section{Acknowledgement}

We acknowledge the CINECA award HP10CX7ERO under the ISCRA initiative, for the availability of high performance computing resources and support. CB gratefully acknowledges financial support by DSSCX project (PRIN 2010-2011, 20104XET32) from Ministero delIIstruzione, dellUniversit e della Ricerca and the University of Torino (Ricerca Locale ex-60\%, Bando 2012). SE thanks MIUR for partial financial support of his Research grant. RB acknowledges financial support by the ImPACT project (FIRB 2012, RBF12CLQD-002) from Ministero dellIstruzione, dellUniversit e della Ricerca. 


\section{References}

[1] V. May and O. Kühn, Charge and Energy Transfer Dynamics in Molecular Systems, Wiley-VCH, Weinheim, 2004.

[2] F. Würthner, G. Archetti, R. Schmidt and H. Kuball, Angew. Chem., Int. Ed., 2008, 47, $4529-4532$.

[3] S. R. Forrest, Nature, 2004, 428, 911-918.

[4] B. O’Regan and M. Grätzel, Nature, 1991, 353, 737-740.

[5] R. Borrelli and A. Peluso, J. Chem. Phys., 2003, 119, 8437-8448.

[6] R. Borrelli and A. Peluso, J. Chem. Phys., 2008, 128, 044303-7.

[7] A. Capobianco, R. Borrelli, C. Noce and A. Peluso, Theor. Chem. Acc., 2012, 131, 1181.

[8] R. Borrelli, A. Capobianco and A. Peluso, J. Phys. Chem. A., 2012, 116, 9934-9940.

[9] R. Borrelli and A. Peluso, J. Chem. Phys., 2006, 125, 194308-194315.

[10] R. Borrelli and A. Peluso, Wiley Interdisciplinary Reviews: Computational Molecular Science, 2013.

[11] F. J. A. Ferrer and F. Santoro, Phys. Chem. Chem. Phys., 2012, 14, 13549-13563.

[12] F. Santoro, A. Lami, R. Improta, J. Bloino and V. Barone, J. Chem. Phys., 2008, 128, 224311-17.

[13] F. Santoro, R. Improta, A. Lami, J. Bloino and V. Barone, J. Chem. Phys., 2007, 126, 084509.

[14] R. Improta, V. Barone and F. Santoro, J. Phys. Chem. B, 2007, 111, 14080-14082.

[15] H.-C. Jankowiak, J. L. Stuber and R. Berger, J. Chem. Phys., 2007, 127, 234101-23.

[16] J. Huh and R. Berger, Faraday Discuss., 2011, 150, 363-373.

[17] R. Berger, C. Fischer and M. Klessinger, J. Phys. Chem. A, 1998, 102, 7157-7167. 
[18] Y. Niu, Q. Peng, C. Deng, X. Gao and Z. Shuai, J. Phys. Chem. A, 2010, 114, 7817-7831.

[19] Q. Peng, Y. Yi, Z. Shuai and J. Shao, J. Chem. Phys., 2007, 126, 114302-8.

[20] Q. Peng, Y. Yi, Z. Shuai and J. Shao, J. Am. Chem. Soc., 2007, 129, 9333-9339.

[21] M. Dierksen and S. Grimme, J. Chem. Phys., 2005, 122, 244101-9.

[22] R. Borrelli, A. Capobianco and A. Peluso, Can. J. Chem., 2013, 91, 495-504.

[23] M. Dierksen and S. Grimme, J. Phys. Chem. A, 2004, 108, 10225-10237.

[24] B. Champagne, M. Guillaume and F. Zutterman, Chem. Phys. Lett., 2006, 425, 105-109.

[25] M. Guillaume, V. Liégeois, B. Champagne and F. Zutterman, Chem. Phys. Lett., 2007, 446, 165-169.

[26] N. De Mitri, S. Monti, G. Prampolini and V. Barone, J. Chem. Theory Comput., 2013.

[27] K. Y. Law, J. Phys. Chem., 1987, 91, 5184-5193.

[28] K.-Y. Law, J. Phys. Chem., 1995, 99, 9818-9824.

[29] P. V. Kamat, S. Das, K. G. Thomas and M. V. George, J. Phys. Chem., 1992, 96, 195-199.

[30] R. W. Bigelow and H. Freund, Chem. Phys., 1986, 107, 159-174.

[31] C. W. Dirk, W. C. Herndon, F. Cervantes-Lee, H. Selnau, S. Martinez, P. Kalamegham, A. Tan, G. Campos and M. Velez, J. Am. Chem. Soc., 1995, 117, 2214-2225.

[32] A. Ajayaghosh, Acc. Chem. Res., 2005, 38, 449-459.

[33] F. C. Law, K. Y.; Bailey, J. Imaging Sci., 1987, 31, 172.

[34] R. J. Meiz, R. B. Champ, L. S. Chang, C. Chiou, C. S. Keller, L. C. Liclican, R. B. Neiman, M. D. Shattuck and W. J. Weiche, Photogr. Sci. Eng., 1977, 21, 73.

[35] C. R. Jipson, V. P.; Jones, IBM Technol. Bull., 1981, 24, 298. 
[36] D. L. Morel, A. K. Ghosh, T. Feng, E. L. Stogryn, P. E. Purwin, R. F. Shaw and C. Fishman, Appl. Phys. Lett., 1978, 32, 495-497.

[37] V. Y. Merritt and H. J. Hovel, Appl. Phys. Lett., 1976, 29, 414-415.

[38] C. Benzi, C. A. Bertolino, I. Miletto, P. Ponzio, C. Barolo, G. Viscardi, S. Coluccia and G. Caputo, Dyes and Pigments, 2009, 83, 111-120.

[39] J. Park, C. Barolo, F. Sauvage, N. Barbero, C. Benzi, P. Quagliotto, S. Coluccia, D. D. Censo, M. Grätzel, M. K. Nazeeruddin and G. Viscardi, Chem. Commun., 48, 2782-2784.

[40] L. Beverina, R. Ruffo, C. M. Mari, G. A. Pagani, M. Sassi, F. De Angelis, S. Fantacci, J.-H. Yum, M. Grätzel and M. K. Nazeeruddin, ChemSusChem, 2009, 2, 621-624.

[41] S. Martiniani, A. Y. Anderson, C. Law, B. C. O'Regan and C. Barolo, Chem. Commun., 2012, 48, 2406-2408.

[42] C. Magistris, S. Martiniani, N. Barbero, J. Park, C. Benzi, A. Anderson, C. Law, C. Barolo and B. O'Regan, Renewable Energy, 2013, 60, 672-678.

[43] K. Sayama, S. Tsukagoshi, T. Mori, K. Hara, Y. Ohga, A. Shinpou, Y. Abe, S. Suga and H. Arakawa, Sol. Energy Mater. Sol. Cells, , 2003, 80, 47-71.

[44] S. Alex, U. Santhosh and S. Das, Journal of Photochemistry and Photobiology, A. Chemistry, 2005, 172, 63-71.

[45] D. Rocca, R. Gebauer, F. De Angelis, M. K. Nazeeruddin and S. Baroni, Chem. Phys. Lett., 2009, 475, 49-53.

[46] J. R. Johnson, N. Fu, E. Arunkumar, W. M. Leevy, S. T. Gammon, D. Piwnica-Worms and B. D. Smith, Angew. Chem., Int. Ed., 2007, 46, 5528-5531.

[47] E. Arunkumar, N. Fu and B. D. Smith, Chemistry: A European Journal, 2006, 12, 4684 4690.

[48] C. Gude and W. Rettig, J. Phys. Chem. A, 2000, 104, 8050-8057. 
[49] G. d. Miguel, M. Marchena, M. Zitnan, S. S. Pandey, S. Hayase and A. Douhal, Phys. Chem. Chem. Phys., 2012, 14, 1796-1805.

[50] G. d. Miguel, M. Marchena, M. Ziek, S. S. Pandey, S. Hayase and A. Douhal, J. Phys. Chem. C, 2012, 116, 12137-12148.

[51] N. Barbero, C. Magistris, J. Park, R. Buscaino, C. Barolo and G. Viscardi, submitted, 2013.

[52] R. Kubo and Y. Toyozawa, Prog. Theor. Phys., 1955, 13, 160-182.

[53] R. Borrelli and A. Peluso, Phys. Chem. Chem. Phys., 2011, 13, 4420-4426.

[54] J. Tang, M. T. Lee and S. H. Lin, J. Chem. Phys., 2003, 119, 7188-7196.

[55] A. Baiardi, J. Bloino and V. Barone, J. Chem. Theory Comput., 2013, 9, 4097-4115.

[56] R. Ianconescu and E. Pollak, J. Phys. Chem. A, 2004, 108, 7778-7784.

[57] M. Lax, J. Chem. Phys., 1952, 20, 1752-1760.

[58] R. Borrelli, M. Thoss, H. Wang and W. Domcke, Mol. Phys., 2012, 110, 751-763.

[59] C. Adamo and V. Barone, J. Chem. Phys., 1999, 110, 6158-6170.

[60] M. J. Frisch, G. W. Trucks, H. B. Schlegel, G. E. Scuseria and M. A. R. et al., Gaussian 09 Revision C.01, Gaussian Inc. Wallingford CT 2009.

[61] R. Borrelli and W. Domcke, Chem. Phys. Lett., 2010, 498, 230-234.

[62] T. E. Sharp and K. M. Rosenstock, J. Chem. Phys., 1964, 41, 3453-3463.

[63] J. R. Reimers, J. Chem. Phys., 2001, 115, 9103-9109.

[64] R. Borrelli and A. Peluso, MolFC: A program for Franck-Condon integrals calculation. Package available online at http: / / www . theochem. unisa.it.

[65] J. Fabian and A. Mehlhorn, Journal of Molecular Structure: THEOCHEM, 1984, 109, $17-26$. 
[66] A. B. Myers, Ann. Rev. Phys. Chem., 1998, 49, 267-295.

[67] P. R. Bunker and P. Jensen, Molecular Symmetry and Spectroscopy, NRC Research Press, Ottawa, 1998.

[68] F. J. Avila Ferrer, J. Cerezo, E. Stendardo, R. Improta and F. Santoro, J. Chem. Theory Comput., 2013, 9, 2072-2082.

[69] F. Momicchioli, A. S. Tatikolov, D. Vanossi and G. Ponterini, Photochemical \& Photobiological Sciences, 2004, 3, 396-402.

[70] R. Schneider and W. Domcke, Chem. Phys. Lett., 1988, 150, 235.

[71] W. W. Parson, Modern Optical Spectroscopy With Exercises and Examples from Biophysics and Biochemistry, Springer Berlin Heidelberg, 2007. 
Table 1 Experimental extinction coefficients (as $\mathrm{M}^{-1} \mathrm{~cm}^{-1}$ ), absorption and emission maxima and their theoretical values computed at PBE0/6-31+G(d,p) level. ${ }^{71}$

\begin{tabular}{cccccc}
$\lambda_{\mathrm{abs}}^{\max }(\mathrm{nm})$ & $\lambda_{\mathrm{em}}^{\max }(\mathrm{nm})$ & $\varepsilon_{\max }$ & $\varepsilon_{\max }^{\mathrm{ISQ6}}$ & $\varepsilon_{\max }^{\mathrm{ISQ} 4}$ & $\varepsilon_{\max }^{\mathrm{av}}$ \\
\hline 659 & 671 & 229000 & 237000 & 137000 & $197000^{a}$ \\
\hline
\end{tabular}

${ }^{a}$ The average value is computed using the Boltzmann populations of the two isomers.

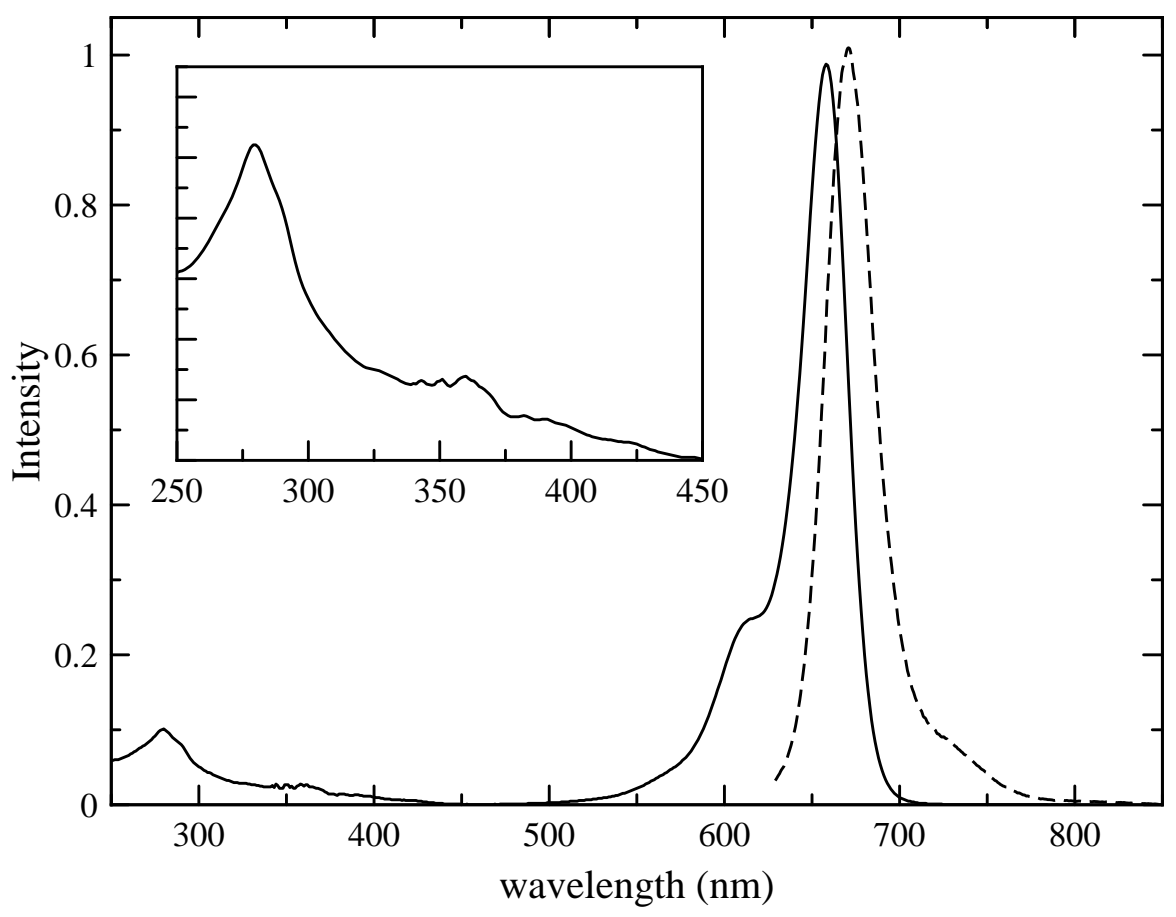

Figure 1 Experimental absorption (full line) and fluorescence (dashed line) spectra of ISQ in tetrahydroduran. Inset: magnification in the region 250-450 nm. 

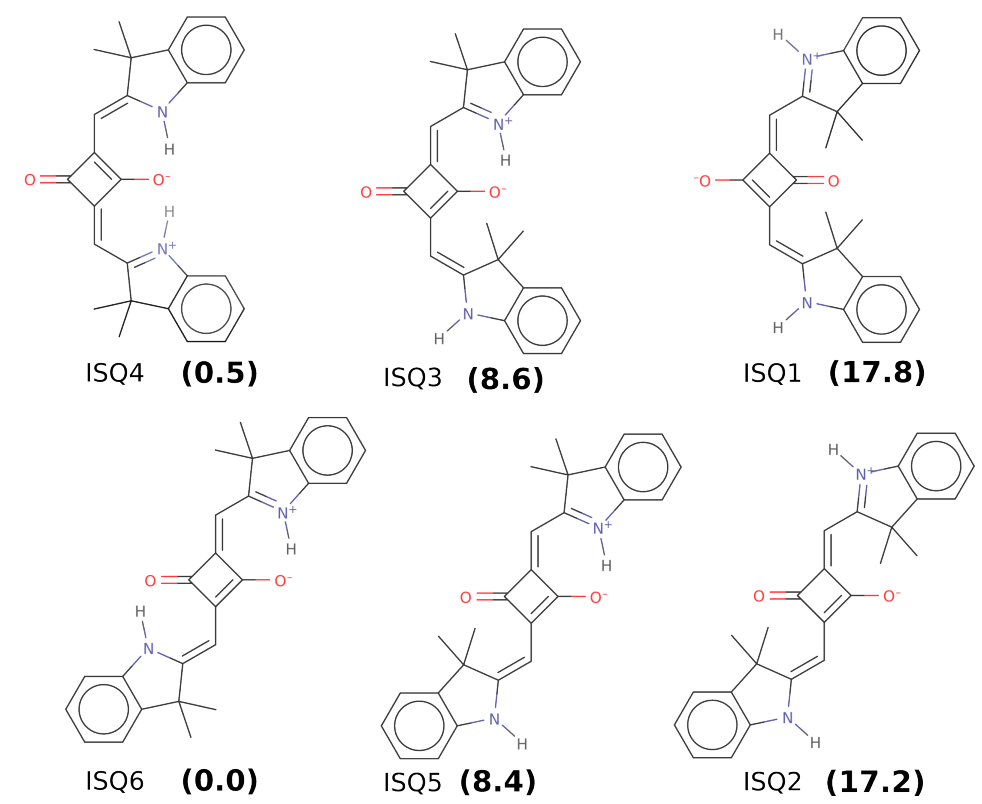

Figure 2 The six most stable indole squaraine isomers and their relative free energies (in $\mathrm{kcal} / \mathrm{mol}$ ) in THF computed at PBE0/PCM level. The labeling proposed by Dirk et al. ${ }^{31}$ has been used. 


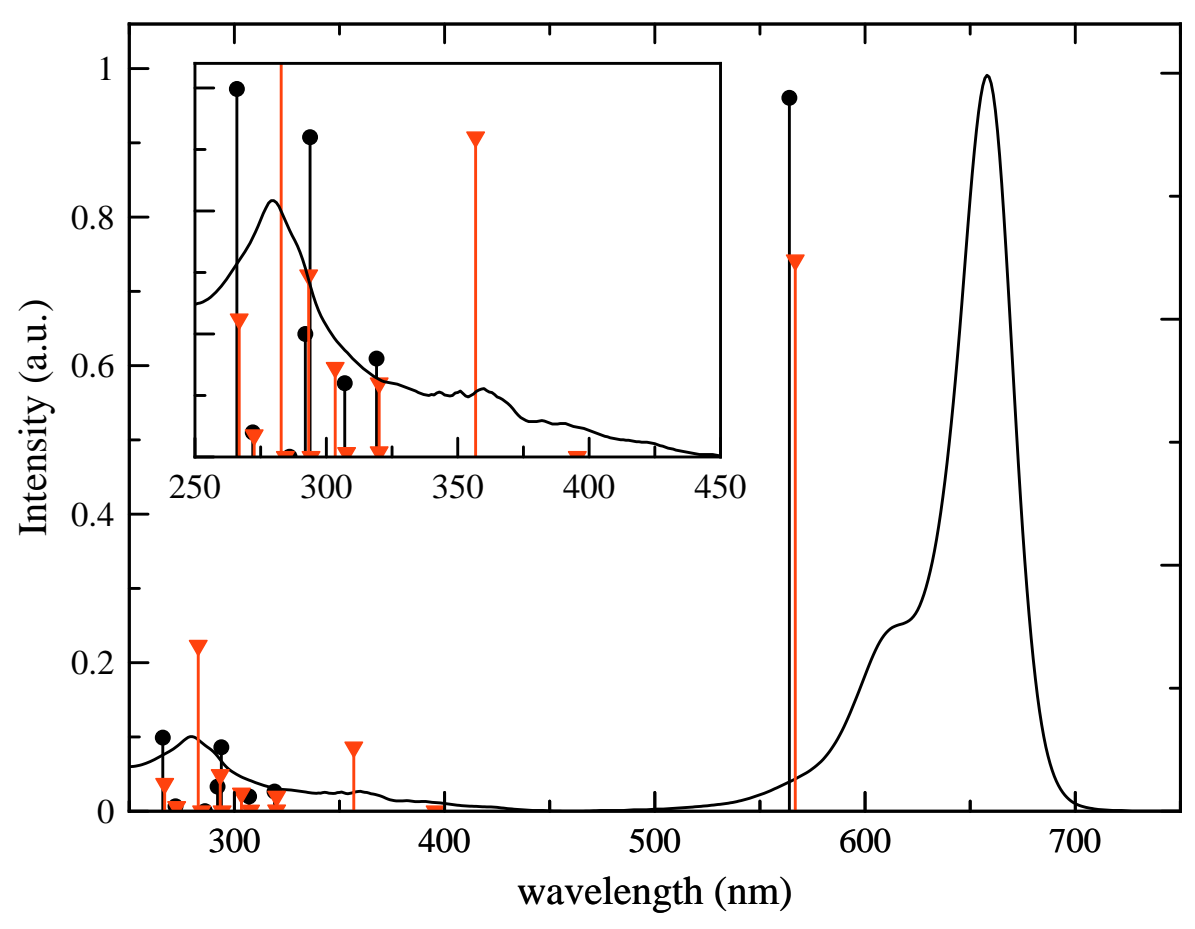

Figure 3 Experimental absorption spectra of ISQ and computed electronic transitions at TD-DFT/PCM level in THF; (•) ISQ6, ( $\mathbf{\nabla})$ ISQ4.

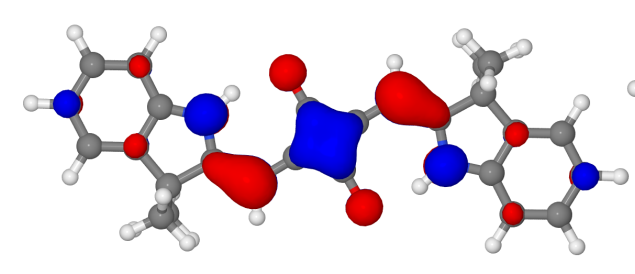

HOMO ISQ6

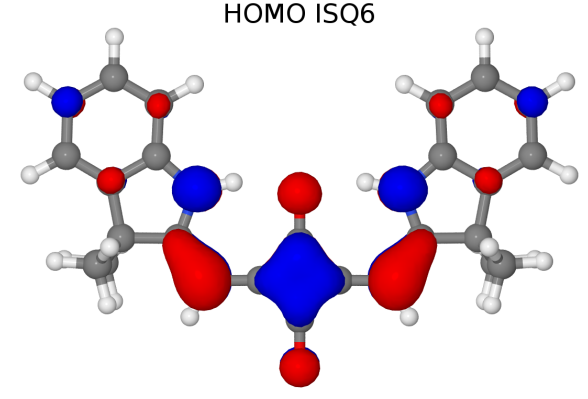

HOMO ISQ4

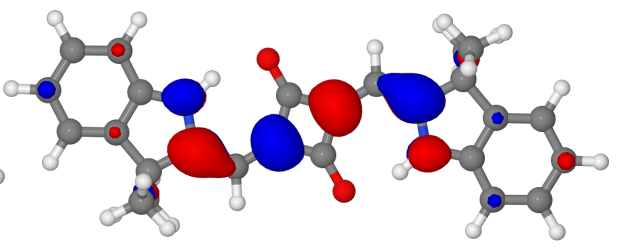

LUMO ISQ6

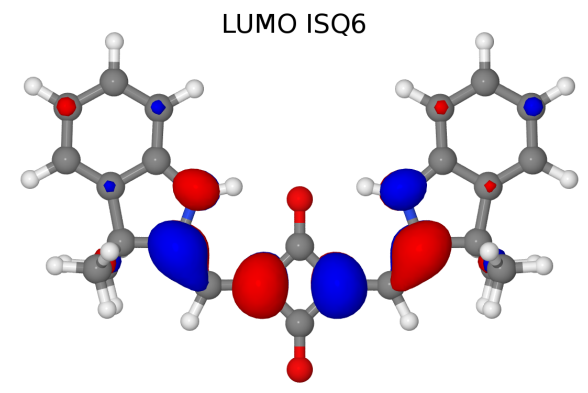

LUMO ISQ4

Figure 4 HOMO and LUMO molecular orbitals involved in the $\mathrm{S}_{1}\left(\pi \pi^{*}\right) \leftarrow \mathrm{S}_{0}$ transition of the isomers ISQ4 and ISQ6. 


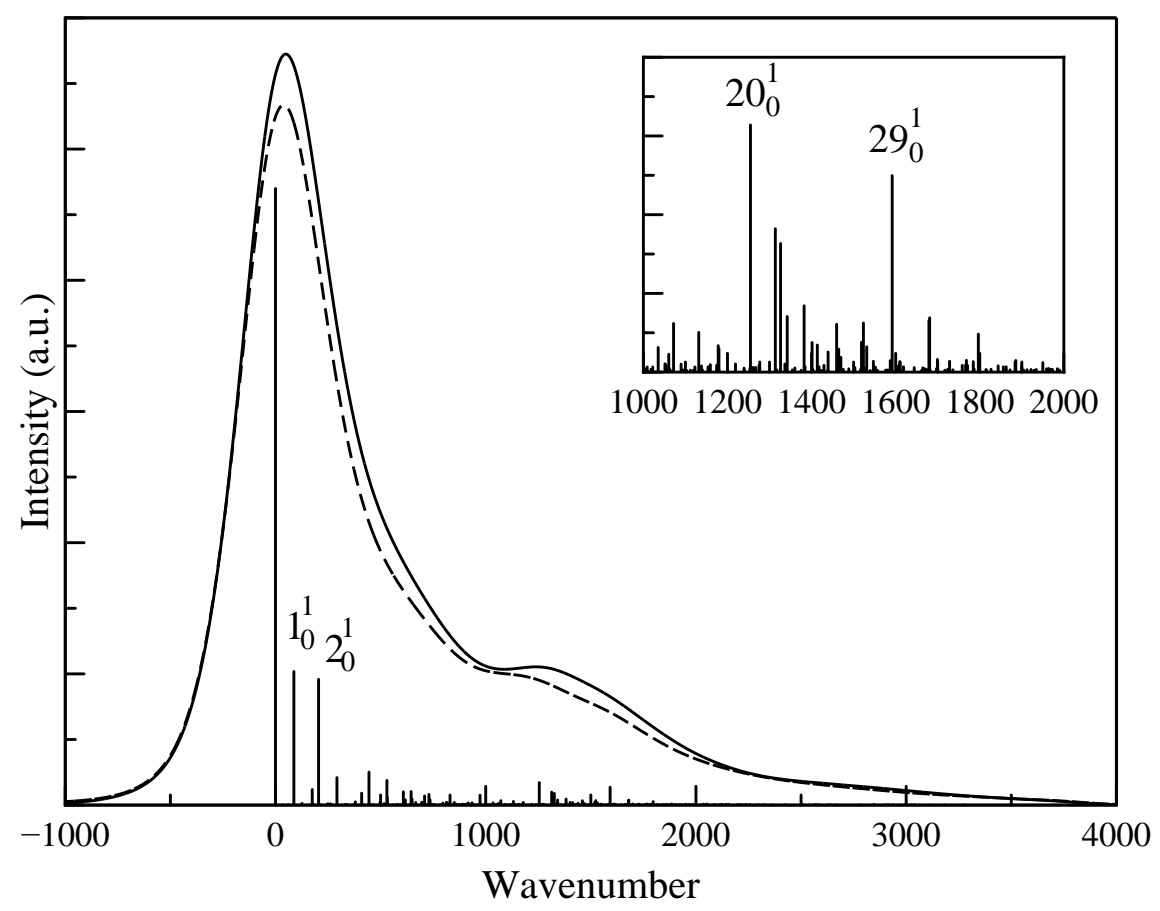

Figure 5 FC absorption spectrum for the $S_{1}\left(\pi \pi^{*}\right) \leftarrow \mathrm{S}_{0}$ transition of ISQ6 at $300 \mathrm{~K}$, computed from DFT/TD-DFT geometries and frequencies. Full line, calculation including bulk solvent effect using PCM; Dashed line, isolated molecule calculation. The 0-0 transition is centered at zero energy. The stick spectrum is obtained at $0 \mathrm{~K}$. 


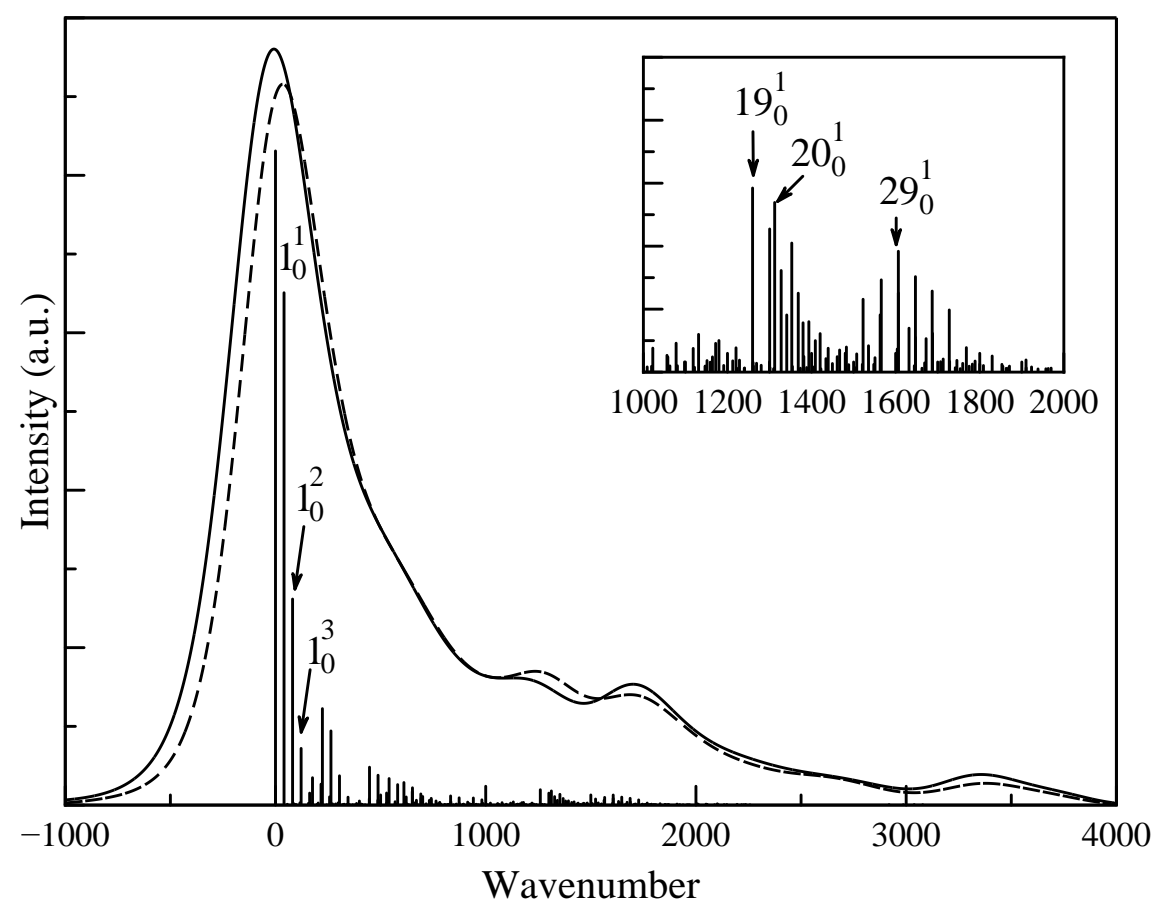

Figure 6 FC absorption spectrum for the $S_{1}\left(\pi \pi^{*}\right) \leftarrow \mathrm{S}_{0}$ transition of ISQ4 at $300 \mathrm{~K}$, computed from DFT/TD-DFT geometries and frequencies. Full line, calculation including bulk solvent effect using PCM; Dashed line, isolated molecule calculation. The 0-0 transition is centered at zero energy. The stick spectrum is obtained at $0 \mathrm{~K}$. 


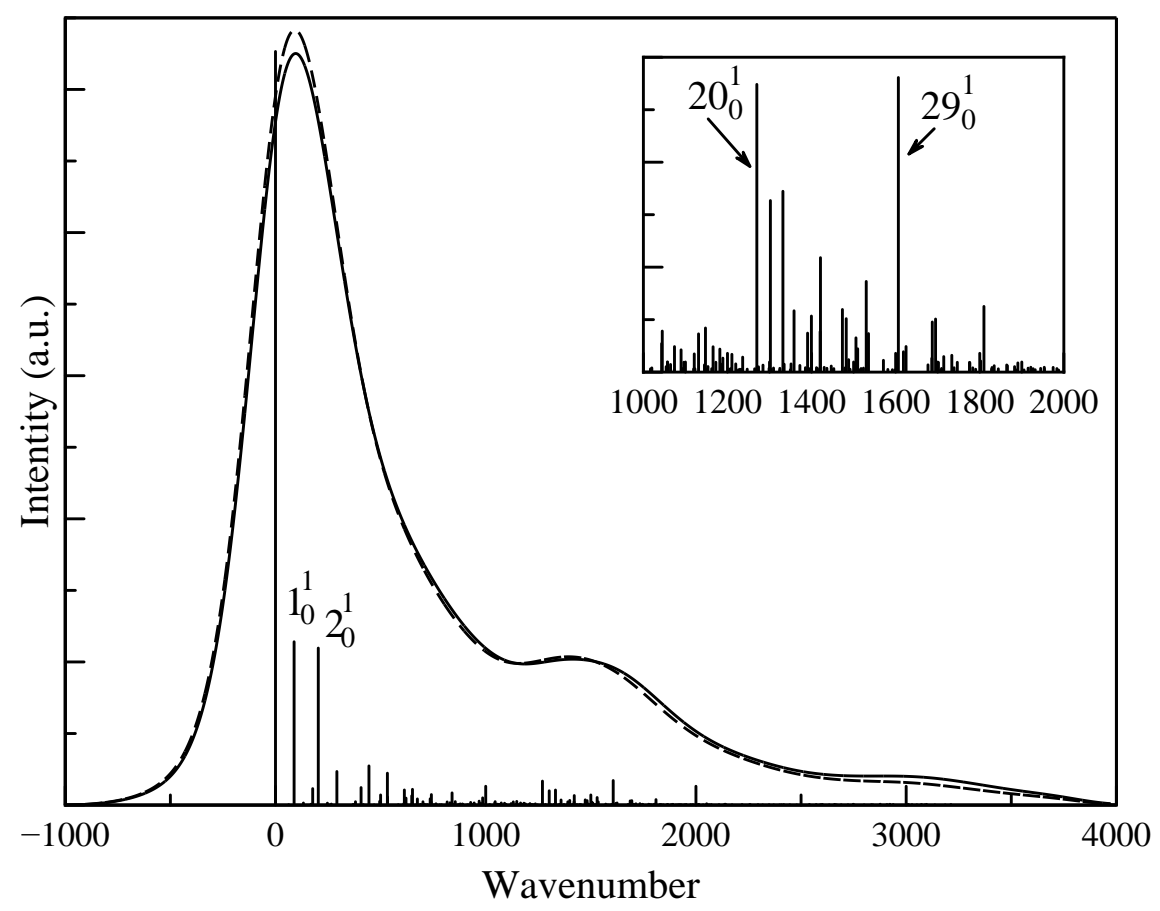

Figure $7 \mathrm{FC}$ emission spectrum for the $S_{1}\left(\pi \pi^{*}\right) \rightarrow \mathrm{S}_{0}$ transition of ISQ6 at $300 \mathrm{~K}$, computed from DFT/TD-DFT geometries and frequencies. Full line, calculation including bulk solvent effect using PCM; Dashed line, isolated molecule calculation. The 0-0 transition is centered at zero energy. The stick spectrum is obtained at $0 \mathrm{~K}$. 


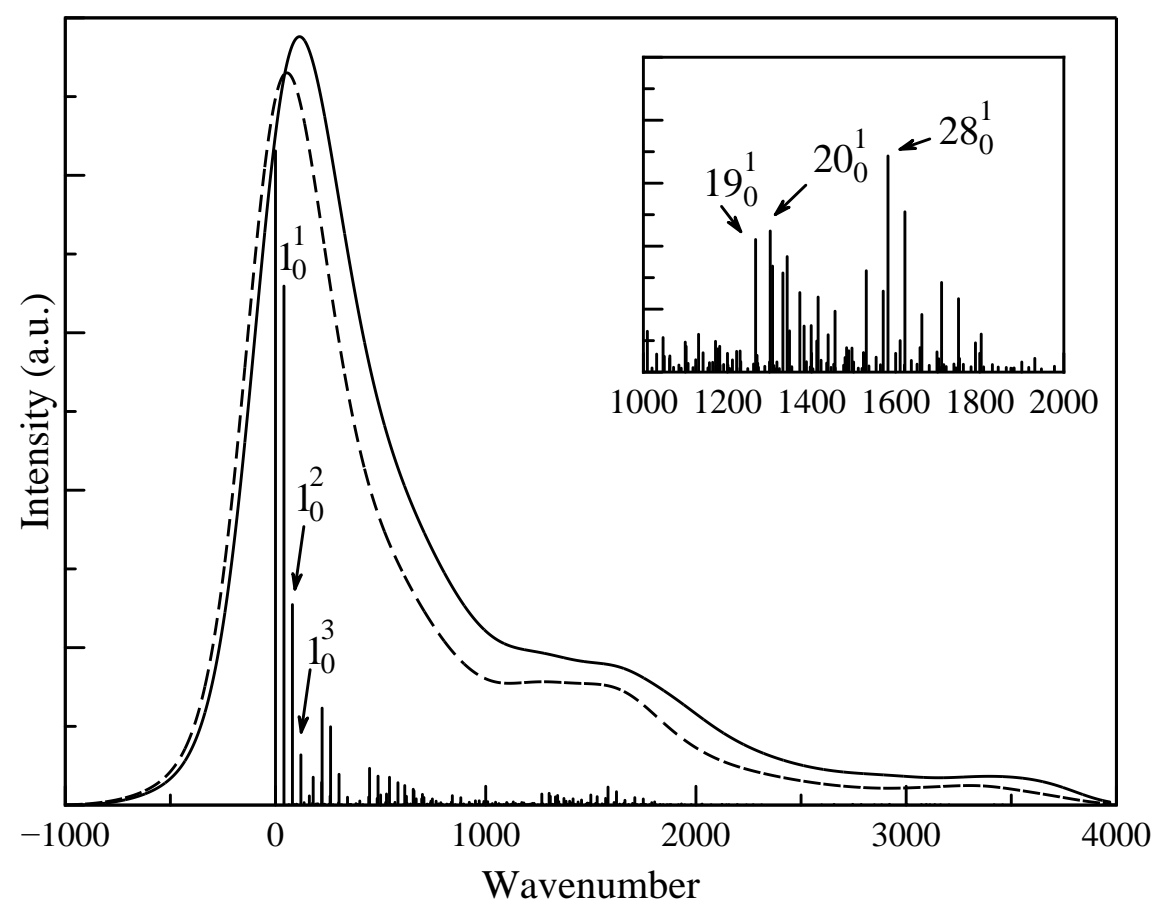

Figure $8 \mathrm{FC}$ emission spectrum for the $S_{1}\left(\pi \pi^{*}\right) \rightarrow \mathrm{S}_{0}$ transition of ISQ4 at $300 \mathrm{~K}$, computed from DFT/TD-DFT geometries and frequencies. Full line, calculation including bulk solvent effect using PCM; Dashed line, isolated molecule calculation. The 0-0 transition is centered at zero energy. The stick spectrum is obtained at $0 \mathrm{~K}$. 


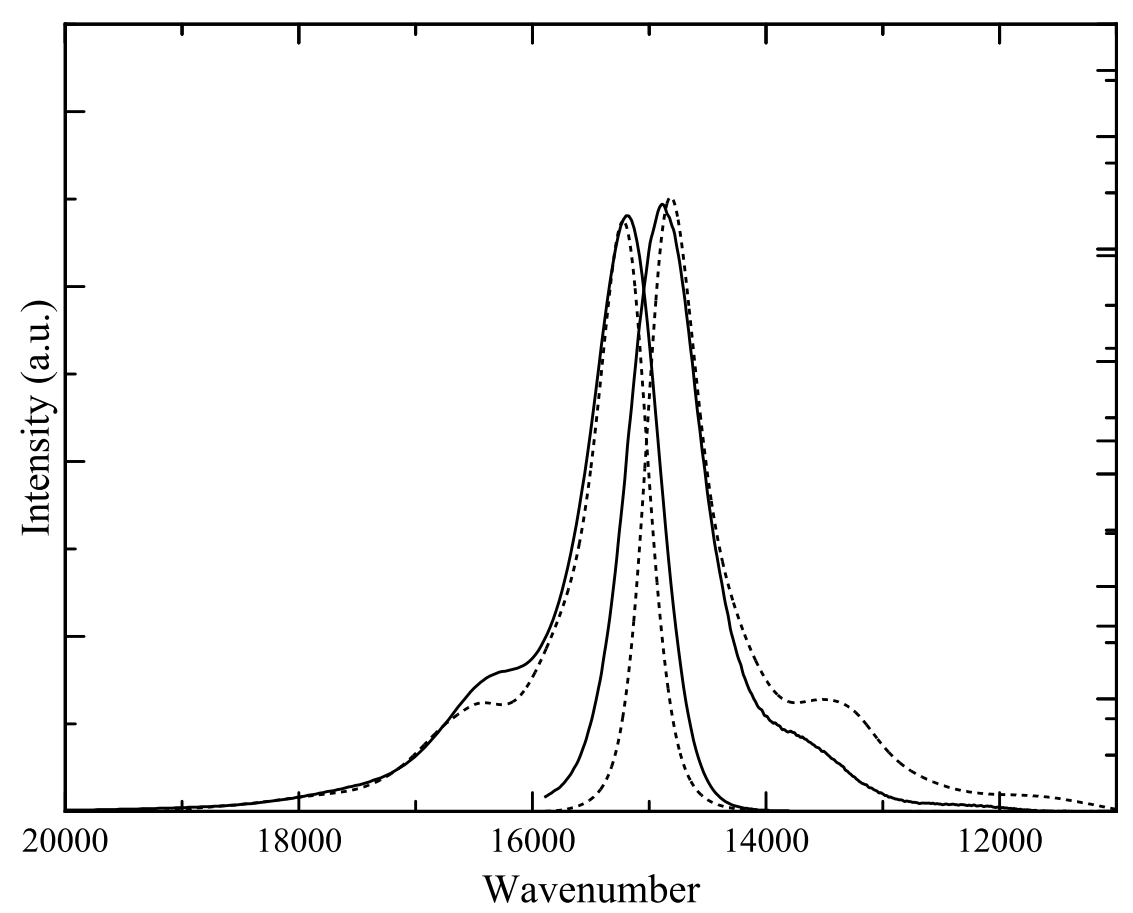

Figure 9 Experimental (full line) and computed (dashed line) absorption and emission spectra of ISQ. Both theoretical and and experimental data are reported as lineshapes. that is the $L_{\mathrm{abs}}(v) \propto \varepsilon(v) / v$ and $L_{\text {emiss }}(v) \propto F(v) / v^{3}$, where $\varepsilon, F$ are the measured absorption and fluorescence intensities. 
Table 2 Vertical excitations energies (expressed as nanometers) of ISQ6 and ISQ4 computed at TD-DFT/6-31+G(d,p) level and TD-DFT/6-31+G(d,p) in tetrahydrofuran. The states are ordered according to their symmetry label. The oscillator strength associated with the transitions are reported in parentheses.

\begin{tabular}{lrr} 
& ISQ6 \\
\hline state & TD-DFT & TD-DFT/PCM \\
$1^{1} B_{u}$ & $538.9(1.26)$ & $564.3(1.45)$ \\
$2^{1} B_{u}$ & $320.9(0.05)$ & $319.1(0.04)$ \\
$3^{1} B_{u}$ & $316.6(0.04)$ & $307.5(0.03)$ \\
$4^{1} B_{u}$ & $287.9(0.04)$ & $293.8(0.13)$ \\
$5^{1} B_{u}$ & $278.0(0.14)$ & $292.0(0.05)$ \\
$6^{1} B_{u}$ & $271.6(0.01)$ & $266.0(0.14)$ \\
$1^{1} A_{u}$ & $301.0\left(10^{-4}\right)$ & $286.8\left(10^{-4}\right)$ \\
$2^{1} A_{u}$ & $275.7(0.01)$ & $272.1(0.01)$ \\
& ISQ4 & \\
\hline state $^{1}$ & TD-DFT & TD-DFT/PCM \\
$1^{1} B_{2}$ & $542.5(0.96)$ & $566.8(1.12)$ \\
$2^{1} B_{2}$ & $322.7(0.015)$ & $320.1(0.002)$ \\
$3^{1} B_{2}$ & $318.5(0.002)$ & $307.7(0.002)$ \\
$4^{1} B_{2}$ & $287.2(0.0)$ & $294.2\left(10^{-4}\right)$ \\
$5^{1} B_{2}$ & $273.8 .7(0.054)$ & $266.8(0.056)$ \\
$1^{1} B_{1}$ & $440.2\left(10^{-4}\right)$ & $395.5\left(10^{-4}\right)$ \\
$2^{1} B_{1}$ & $296.0(0.0)$ & $284.4\left(10^{-4}\right)$ \\
$3^{1} B_{1}$ & $277.0(0.009)$ & $272.5(0.009)$ \\
$1^{1} A_{1}$ & $352.1(0.07)$ & $356.8(0.13)$ \\
$2^{1} A_{1}$ & $326.7(0.01)$ & $320.1(0.03)$ \\
$3^{1} A_{1}$ & $304.6(0.15)$ & $303.4(0.04)$ \\
$4^{1} A_{1}$ & $286.8(0.05)$ & $293.3(0.07)$ \\
$5^{1} A_{1}$ & $271.2(0.13)$ & $282.8(0.34)$
\end{tabular}


Table 3 Dimensionless displacement vector $\mathbf{K}$ associated to the $S_{1} \leftarrow S_{0}$ transition of ISQ4 in vacuo, and in THF. Solvent effects are taken into account using PCM with $\varepsilon=7.42$. High frequency vibrations have a negligible displacement and are not reported. Vibrations are numbered according to their vibrational frequency.

\begin{tabular}{l|cc|r|rc|r} 
& \multicolumn{3}{|c|}{ ISQ4 in vacuo } & \multicolumn{3}{c}{ ISQ4 in THF } \\
\hline & $\omega\left(S_{1}\right)$ & $\omega\left(S_{0}\right)$ & $\mathrm{K}$ & $\omega\left(S_{1}\right)$ & $\omega\left(S_{0}\right)$ & $\mathrm{K}$ \\
\hline 1 & 41 & 40 & 1.262 & 39 & 38 & 1.097 \\
2 & 176 & 179 & 0.289 & 176 & 179 & 0.312 \\
3 & 223 & 222 & -0.533 & 221 & 219 & -0.579 \\
4 & 447 & 448 & 0.337 & 447 & 447 & 0.319 \\
5 & 540 & 542 & -0.286 & 539 & 541 & -0.324 \\
6 & 611 & 615 & 0.258 & 610 & 615 & 0.267 \\
7 & 651 & 659 & -0.210 & 655 & 659 & -0.123 \\
8 & 700 & 706 & 0.150 & 701 & 706 & 0.171 \\
9 & 765 & 775 & -0.053 & 763 & 773 & -0.029 \\
10 & 833 & 841 & -0.167 & 834 & 842 & -0.171 \\
11 & 891 & 892 & 0.042 & 892 & 891 & 0.038 \\
12 & 941 & 951 & 0.140 & 943 & 952 & 0.155 \\
13 & 1051 & 1054 & 0.026 & 1049 & 1052 & 0.030 \\
14 & 1078 & 1102 & 0.085 & 1084 & 1105 & 0.079 \\
15 & 1131 & 1131 & 0.097 & 1130 & 1129 & 0.101 \\
16 & 1180 & 1182 & 0.089 & 1177 & 1179 & 0.105 \\
17 & 1198 & 1201 & -0.022 & 1199 & 1201 & -0.043 \\
18 & 1229 & 1232 & -0.053 & 1231 & 1233 & -0.065 \\
19 & 1260 & 1267 & 0.213 & 1261 & 1269 & 0.214 \\
20 & 1312 & 1302 & 0.204 & 1310 & 1294 & 0.254 \\
21 & 1328 & 1332 & -0.158 & 1329 & 1333 & -0.171 \\
22 & 1380 & 1399 & -0.104 & 1386 & 1396 & -0.171 \\
23 & 1402 & 1416 & 0.024 & 1400 & 1411 & 0.044 \\
24 & 1440 & 1436 & 0.080 & 1425 & 1427 & 0.048 \\
25 & 1467 & 1483 & -0.071 & 1464 & 1475 & -0.066 \\
26 & 1517 & 1525 & -0.010 & 1514 & 1519 & -0.109 \\
27 & 1522 & 1530 & -0.136 & 1518 & 1526 & 0.133 \\
28 & 1566 & 1582 & -0.155 & 1566 & 1579 & -0.127 \\
29 & 1606 & 1658 & 0.181 & 1615 & 1645 & 0.140 \\
30 & 1632 & 1680 & 0.103 & 1629 & 1668 & 0.080 \\
31 & 1669 & 1703 & 0.049 & 1665 & 1679 & 0.053 \\
32 & 1687 & 1709 & -0.144 & 1681 & 1697 & -0.144 \\
33 & 1821 & 1862 & 0.006 & 1794 & 1829 & -0.065
\end{tabular}


Table 4 Dimensionless displacement vector $\mathbf{K}$ associated to the $S_{1} \leftarrow S_{0}$ transition of ISQ6 in vacuo and in THF. Solvent effects are taken into account using the PCM model with $\varepsilon=7.42$. High frequency vibrations have a negligible displacement and are not reported. Vibrations are numbered according to their vibrational frequency.

\begin{tabular}{l|rr|r|rc|r} 
& \multicolumn{3}{|c|}{ ISQ6 in vacuo } & \multicolumn{3}{|c}{ ISQ6 in THF } \\
\hline & $\omega\left(S_{1}\right)$ & $\omega\left(S_{0}\right)$ & $\mathrm{K}$ & $\omega\left(S_{1}\right)$ & $\omega\left(S_{0}\right)$ & $\mathrm{K}$ \\
\hline 1 & 87 & 88 & 0.665 & 84 & 85 & -0.590 \\
2 & 205 & 204 & -0.633 & 204 & 202 & 0.703 \\
3 & 251 & 248 & 0.061 & 239 & 238 & -0.046 \\
4 & 445 & 445 & 0.323 & 442 & 442 & 0.304 \\
5 & 530 & 532 & 0.286 & 529 & 532 & -0.335 \\
6 & 608 & 613 & 0.206 & 607 & 612 & 0.226 \\
7 & 645 & 652 & -0.213 & 645 & 649 & 0.132 \\
8 & 667 & 675 & 0.099 & 668 & 674 & 0.107 \\
9 & 730 & 742 & -0.181 & 731 & 741 & -0.162 \\
10 & 830 & 840 & 0.178 & 830 & 840 & 0.162 \\
11 & 858 & 870 & 0.057 & 858 & 870 & -0.100 \\
12 & 889 & 892 & -0.051 & 891 & 892 & 0.061 \\
13 & 973 & 985 & 0.171 & 970 & 984 & 0.169 \\
14 & 1051 & 1054 & -0.048 & 1048 & 1051 & -0.049 \\
15 & 1072 & 1131 & 0.110 & 1060 & 1129 & -0.064 \\
16 & 1132 & 1148 & 0.107 & 1130 & 1150 & 0.105 \\
17 & 1180 & 1182 & 0.082 & 1175 & 1177 & -0.100 \\
18 & 1197 & 1205 & 0.022 & 1196 & 1204 & 0.005 \\
19 & 1227 & 1233 & -0.007 & 1227 & 1233 & -0.024 \\
20 & 1255 & 1270 & 0.265 & 1255 & 1270 & -0.256 \\
21 & 1314 & 1302 & 0.203 & 1310 & 1294 & -0.255 \\
22 & 1326 & 1332 & 0.190 & 1326 & 1332 & 0.183 \\
23 & 1382 & 1400 & 0.131 & 1387 & 1395 & -0.184 \\
24 & 1401 & 1421 & 0.030 & 1399 & 1413 & -0.049 \\
25 & 1439 & 1436 & -0.079 & 1426 & 1429 & -0.046 \\
26 & 1465 & 1482 & -0.075 & 1461 & 1473 & 0.067 \\
27 & 1517 & 1523 & 0.033 & 1513 & 1519 & 0.119 \\
28 & 1523 & 1530 & -0.120 & 1519 & 1525 & 0.112 \\
29 & 1592 & 1606 & 0.244 & 1585 & 1603 & -0.175 \\
30 & 1610 & 1669 & -0.064 & 1616 & 1661 & 0.106 \\
31 & 1666 & 1687 & -0.032 & 1662 & 1678 & -0.038 \\
32 & 1681 & 1702 & -0.128 & 1676 & 1693 & 0.134 \\
33 & 1783 & 1829 & 0.022 & 1777 & 1818 & 0.083
\end{tabular}

